\title{
What Happens to Apparent Horizons in a Binary Black Hole Merger?
}

\author{
Daniel Pook-Kolb, ${ }^{1,2}$ Robie A. Hennigar, ${ }^{3,4,5}$ and Ivan Booth ${ }^{3}$ \\ ${ }^{1}$ Max-Planck-Institut für Gravitationsphysik (Albert Einstein Institute), Callinstr. 38, 30167 Hannover, Germany \\ ${ }^{2}$ Leibniz Universität Hannover, 30167 Hannover, Germany \\ ${ }^{3}$ Department of Mathematics and Statistics, Memorial University, \\ St. John's, Newfoundland and Labrador, A1C 5S7, Canada \\ ${ }^{4}$ Department of Physics and Astronomy, University of Waterloo, Waterloo, Ontario, Canada, N2L $3 G 1$ \\ ${ }^{5}$ Department of Physics and Computer Science, Wilfrid Laurier University, Waterloo, Ontario, Canada N2L $3 C 5$
}

\begin{abstract}
We resolve the fate of the two original apparent horizons during the head-on merger of two non-spinning black holes. We show that following the appearance of the outer common horizon and subsequent interpenetration of the original horizons, they continue to exist for a finite period of time before they are individually annihilated by unstable MOTSs. The inner common horizon vanishes in a similar, though independent, way. This completes the understanding of the analogue of the event horizon's "pair of pants" diagram for the apparent horizon. Our result is facilitated by a new method for locating marginally outer trapped surfaces (MOTSs) based on a generalized shooting method. We also discuss the role played by the MOTS stability operator in discerning which among a multitude of MOTSs should be considered as black hole boundaries.
\end{abstract}

It is common practice to picture the merger of two black holes according to the famous "pair of pants" diagram, which describes the evolution of the event horizon during the merger. But what does the analogous picture for the apparent horizon $(\mathrm{AH})$ look like? While the evolution of the event horizon has been understood for nearly half a century [1, 2], the complete evolution of apparent horizons during a merger has remained unresolved.

While the event horizon is well-suited to theoretical analyses, its teleological nature makes it less useful in highly dynamical or practical situations. In numerical simulations of mergers, marginally outer trapped surfaces (MOTSs) are used instead. A closed two-dimensional space-like surface $\mathcal{S}$ is a MOTS if light rays emitted normal to $\mathcal{S}$ are neither converging nor diverging in the outward direction. The outermost MOTS on a given slice is commonly called the apparent horizon. However, despite their importance as a quasi-local characterization of black holes, there remain many unresolved questions pertaining to the evolution of interior MOTSs during a merger.

While it is true that the details of what occurs within the event horizon (where all MOTSs are located) is causally disconnected from the rest of the universe, this does not mean that interior evolution is irrelevant. At the very least, it is conceptually important to understand to what extent MOTSs provide a physically reasonable description of the merger. Furthermore, the existence of a connected sequence of MOTSs between the initial and final states of the merger provides a means for physical properties [3-5] to be tracked throughout the full evolution. Finally, one may hope that there exist correlations between the dynamics in the strong field regime and properties of the distant spacetime. Indeed, such correlations have been shown to exist under certain circumstances [5-9].

The behaviour of AHs during the initial stages of a merger is well-known [1]. Initially there are two individual AHs corresponding to two separate black holes.
When these holes become sufficiently close to one another, a common $\mathrm{AH}$ forms surrounding the individual horizons, which continue to exist. This common horizon immediately splits into an inner and outer branch. The outer branch grows in area and becomes more symmetric, ultimately asymptoting to the event horizon. The inner common MOTS moves inward, becoming increasingly distorted.

The bifurcation of the common horizon, combined with the fact that there are known exact solution examples of MOTSs weaving back and forth through time [10, 11], led to the speculative idea that all MOTSs involved in the merger may be different components of a single world tube that weaves its way through time [12, 13]. However, in most situations of interest MOTSs must be located numerically. Therefore, improvements in the understanding of the evolution of MOTSs during a merger have been in lockstep with improvements in the numerical methods used to locate them. For this reason, progress beyond this qualitative speculation was limited.

With the advent of more robust numerical finders for MOTSs [14], it is now possible to go beyond these initial stages of the merger and better understand the interior dynamics. The inner common MOTS continues to move inward and coincides with the union of the two AHs of the individual black holes at the moment these horizons touch. This can be interpreted as an instantaneous (nonsmooth) merger of the three MOTSs [15--18]. All continue to exist beyond this point: the two individual horizons interpenetrate and the inner common MOTS develops selfintersections. Identifying these self-intersections was not possible with previous MOTS finders, as these had implicitly assumed that the MOTSs are 'star-shaped'. These seemingly exotic surfaces have subsequently been shown to be rather generic. For example, there are an infinite number of self-intersecting MOTSs present within the horizon of the Schwarzschild black hole [19]. This raises the ques- 
tion of whether exotic MOTSs are also present during a black hole merger, and what role they might play.

In this Letter, we report three closely connected results. First, using a novel MOTS finder, we identify for the first time an apparently infinite number of MOTSs present in certain Brill-Lindquist initial data. We then discuss the role that these new MOTSs play in resolving the final fate of the AHs of the two original black holes, finding that there do exist world tubes weaving back and forth through time. However, instead of a single smooth world tube, we find multiple distinct ones. Finally, we discuss the stability of these surfaces. The stability operator characterizes key merger processes and identifies which MOTSs should be thought of as black hole boundaries.

A Multitude of MOTSs. To locate MOTSs, we employ a novel shooting method. The procedure, while in the tradition of methods first developed in the 1970s [20], is more versatile and applies to non-rotating though otherwise general axisymmetric configurations [21]. Implemented in [22], it can be applied to both analytically known initial data as well as to slices obtained from numerical simulations. It overcomes a limitation of the method introduced in [14] as it does not require an initial guess for the shape of the surface to be located. As such, it is ideally suited for locating axisymmetric MOTSs with geometries that are both unexpected and arbitrarily complicated.

The approach exploits axisymmetry to reduce the problem of locating a 2-surface to that of determining a curve $\gamma$, which we call a MOTSodesic. The full MOTS is the revolution of $\gamma$. Given an axisymmetric 3-surface $\left\{\Sigma, h_{i j}, D_{i}\right\}$, the arclength parameterized curve $\gamma(s)$ in the two-dimensional space orthogonal to the rotational Killing field $\varphi=\frac{\partial}{\partial \phi}$ is a MOTSodesic if it satisfies the two coupled second-order ODEs:

$$
T^{a} D_{a} T^{b}=\left(N^{c} D_{c}(\ln R)+k_{u}\right) N^{b} \equiv \kappa^{+} N^{b} .
$$

Here $T^{a}$ is the unit-length tangent vector to $\gamma, N^{a}$ is its unit-length normal, $R$ is the circumferential radius, and $k_{u}$ is the trace of the extrinsic curvature of $\mathcal{S}$ with respect to the unit time-like normal $u$ to $\Sigma$.

Henceforth, we restrict our considerations to the headon merger of two non-spinning black holes. For this purpose, we use Brill-Lindquist (BL) initial data [23]. These describe a Cauchy slice $\Sigma$ which is time symmetric, i.e. with vanishing extrinsic curvature. The three-metric is conformally flat, $h_{i j}=\psi^{4} \delta_{i j}$, where $\delta_{i j}$ is the flat metric and the conformal factor is

$$
\psi=1+\frac{m_{1}}{2 r_{1}}+\frac{m_{2}}{2 r_{2}},
$$

where $m_{1,2}$ are the bare masses of the black holes and $r_{1,2}$ are the (coordinate) distances to the respective punctures.

Parametrizing $\gamma:(\rho, z)=(P(s), Z(s))$ relative to cylindrical coordinates $(\rho, z, \phi)$, the MOTSodesic equa-
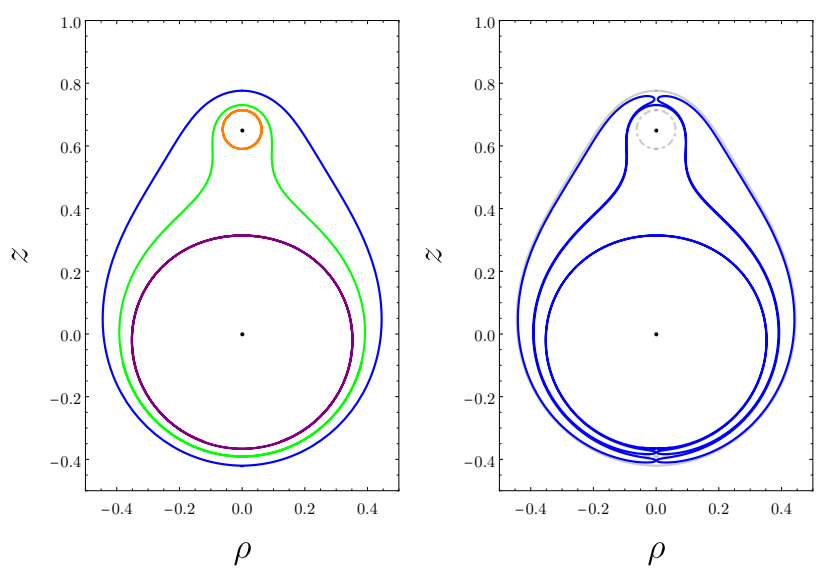

FIG. 1. MOTSs present in Brill-Lindquist initial data for $m_{1}=$ $0.2, m_{2}=0.8$ and separation $d=0.65$. Left: The 'standard' horizons consisting of the outer AH (blue), inner common MOTS (green), and AHs of individual black holes (orange and purple) reproduced using the shooting method. Right: An example of a new exotic MOTS.

tions become

$$
\begin{aligned}
& \ddot{P}=\frac{\dot{Z}^{2}}{P}+\frac{4 \psi_{\rho}}{\psi^{5}}-\frac{6 \dot{P}\left(\dot{P} \psi_{\rho}+\dot{Z} \psi_{z}\right)}{\psi}, \\
& \ddot{Z}=-\frac{\dot{Z} \dot{P}}{P}+\frac{4 \psi_{z}}{\psi^{5}}-\frac{6 \dot{Z}\left(\dot{P} \psi_{\rho}+\dot{Z} \psi_{z}\right)}{\psi},
\end{aligned}
$$

where subscripts denote partial derivatives and the arclength parameterization reads $\psi^{4}\left(\dot{P}^{2}+\dot{Z}^{2}\right)=1$. The equations are solved in Mathematica using the shooting method (and the resulting MOTSs confirmed using the methods of [14]).

In addition to the four 'standard' MOTSs, we find a large number of more exotic MOTSs - see Fig. 1 for an example. In BL initial data, all new MOTSs are found between the outer $\mathrm{AH}$ and the two original AHs and can enclose either, both, or neither of the two punctures. These surfaces tend to closely 'hug' the outer and/or the individual AHs but can fold a seemingly arbitrary number of times. By carefully tuning initial conditions, we find more and more MOTSs. The number is likely infinite.

The Fate of the Apparent Horizons. The existence of new MOTSs in BL initial data raises a number of important questions. First, are these surfaces generic during a merger, or are they artefacts of the symmetry of the initial data? Second, if generic, what role (if any) do these surfaces play in the merger? Finally, with a seemingly infinite number of MOTSs present in a merger, how can one discern physically relevant surfaces that demarcate black hole boundaries?

To address these questions, we numerically evolve the initial data. Here we start with total ADM mass $M=$ $m_{1}+m_{2}=1$, mass ratio $q=m_{2} / m_{1}=2$ and distance parameter $d=0.9$, corresponding to two black holes that are initially separate with no common $\mathrm{AH}$ or any of the 


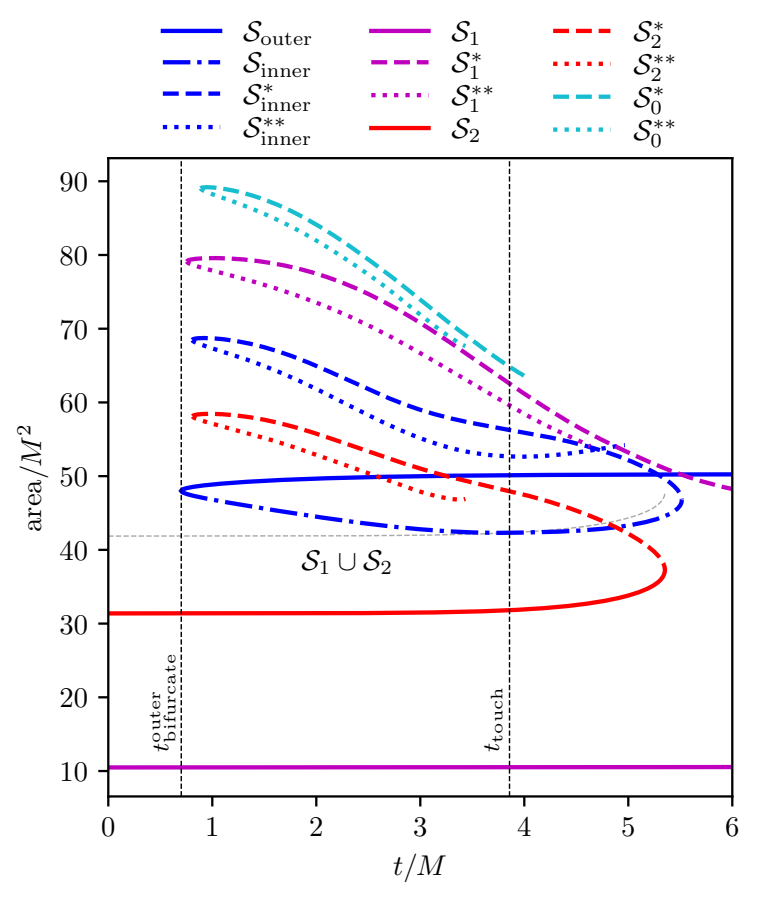

FIG. 2. The area of the various MOTSs as a function of time. Lines of the same colour indicate continuous world tubes moving forward and backward in time. The pale gray dashed line indicates the sum of areas of $\mathcal{S}_{1,2}$. For numerical reasons, we lose track of some of the MOTSs - this is why some of the curves abruptly terminate.

new MOTSs present. To track evolving MOTSs we use the method described in [14, 16] and available from [22]. To locate new MOTSs we use not only the shooting method described earlier (detailed in [21]) but also the ansatz that MOTSs only appear or disappear in pairs. When a MOTS cannot be tracked into the future or past, we look for a "nearby" one with which it might annihilate or bifurcate.

We perform our simulations with the Einstein Toolkit [24, 25] and set up initial conditions using TwoPunctures [26]. The Einstein equations are evolved in the BSSN formulation using an axisymmetric version of McLachlan [27], which uses Kranc [28, 29] to generate C++ code. We work with a $1+\log$ slicing and a $\Gamma$-driver shift condition [30, 31]. Most results are obtained with a spatial grid resolution of $1 / \Delta x=720$. Lower resolutions and shorter simulations with resolutions up to $1 / \Delta x=1920$ are used to assess convergence and resolve certain features (see [16, 32] for more details).

Our main results are illustrated in Fig. 2, which shows the area evolution of several relevant MOTSs, and Fig. 3 which shows those MOTSs at a particular moment of time: $\mathcal{S}_{\text {outer }}$ is the common $\mathrm{AH}, \mathcal{S}_{\text {inner }}$ is the inner common MOTS, and $\mathcal{S}_{1,2}$ correspond to the AHs of the individual black holes. Beyond these standard MOTSs, we find many new MOTSs; a selection appear in Fig. 2 The new sur-

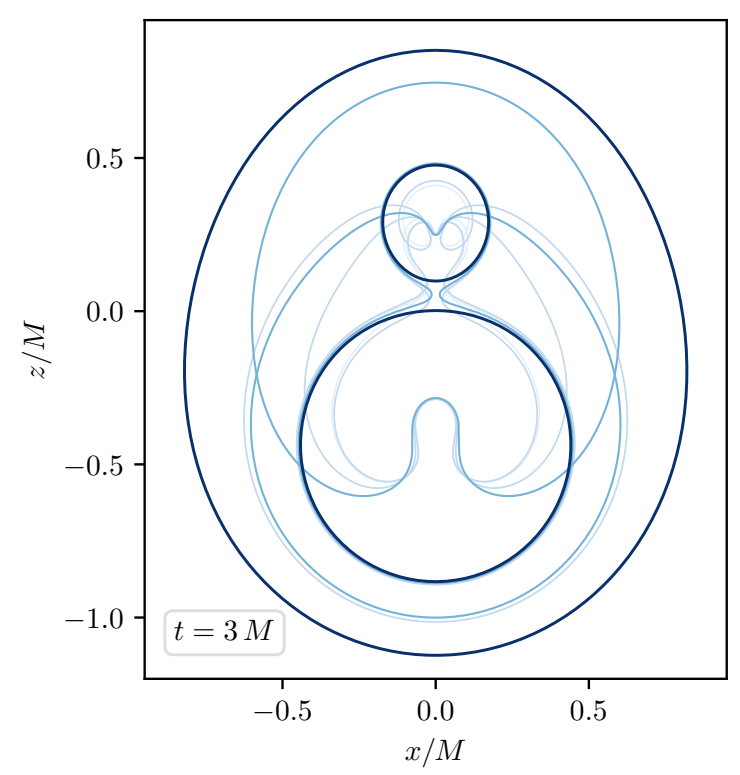

FIG. 3. A "snapshot" of Fig. 2 showing a variety of MOTSs. The three dark lines correspond to $\mathcal{S}_{\text {outer }}$ and $\mathcal{S}_{1,2}$. Two of the shown surfaces exhibit self-intersections. Lighter colors indicate a larger number of negative eigenvalues of the stability operator (see below and [21, 32] for details). Note that, in contrast to BL initial data, the absence of time symmetry means that it is now possible for the new MOTS to penetrate the inner AHs.

faces all form through bifurcations, splitting into outer and inner branches. Hence exotic MOTSs are not solely artefacts of time symmetry. The shown MOTSs all form after the outer AH has formed, and despite several having larger area than $\mathcal{S}_{\text {outer }}$, are all contained within it.

The figure makes clear that exotic MOTSs are essential to understanding the final fate of the AHs of the individual black holes. Both $\mathcal{S}_{\text {inner }}$ and $\mathcal{S}_{2}$ are independently annihilated by new MOTSs. We have good indications that $\mathcal{S}_{1}$ is annihilated by $\mathcal{S}_{1}^{*}$, but this could not be fully resolved in our simulation due to the MOTSs becoming too close to the punctures, where the resolution is necessarily worse. Nonetheless, it seems clear that our new MOTSs provide a mechanism by which the AHs of the original black holes are annihilated. To illustrate this in greater detail, we present in Fig. 4 several "snapshots" of the evolution of $\mathcal{S}_{2}$.

The Role of Stability. The stability operator is key in understanding the details of the picture so far described. For a non-spinning axisymmetric MOTS in vacuum, this takes the form [21]:

$$
L_{\Sigma} \Psi=-\Delta_{\mathcal{S}} \Psi+\left(\frac{\mathcal{R}}{2}-2\left|\sigma_{+}\right|^{2}\right) \Psi,
$$

where $\Delta_{\mathcal{S}}$ is the Laplacian on $\mathcal{S}, \mathcal{R}$ is its scalar curvature, and $\sigma_{+}^{A B}$ is the shear in the outward null direction. Geometrically this operator arises when one considers local deformations of a given MOTS, with the function $\Psi$, 

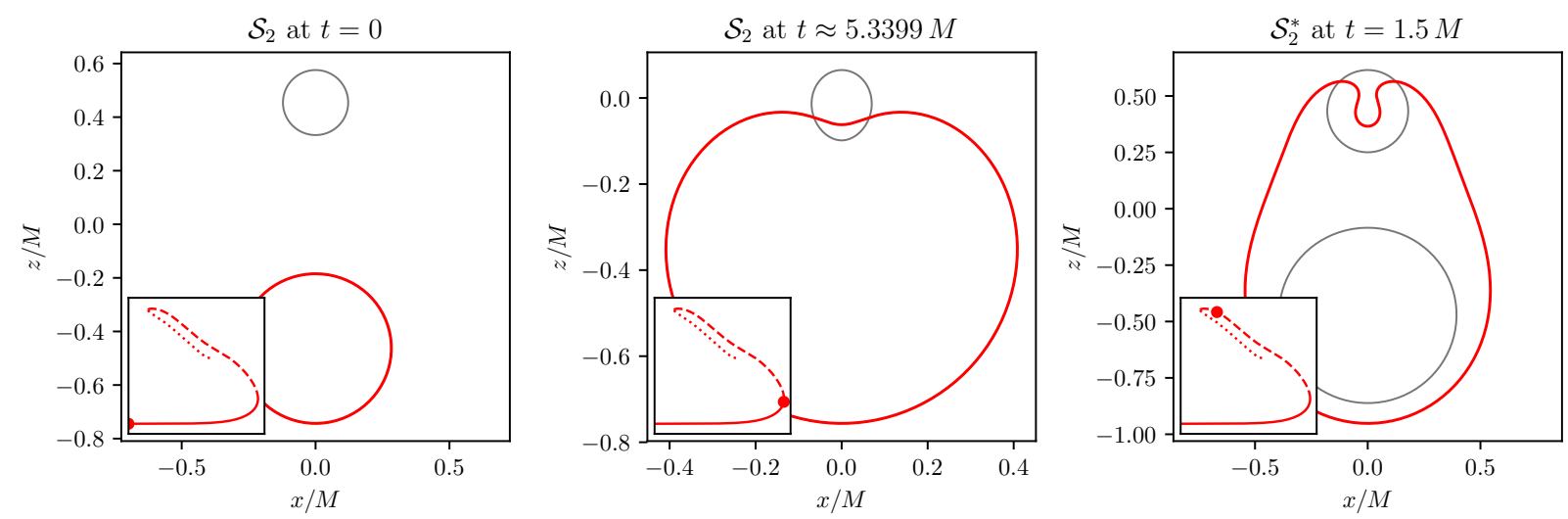

FIG. 4. The annihilation of $\mathcal{S}_{2}$. The inset shows, with a red dot, where along the world tube the shown MOTS occurs [see Fig. 2].

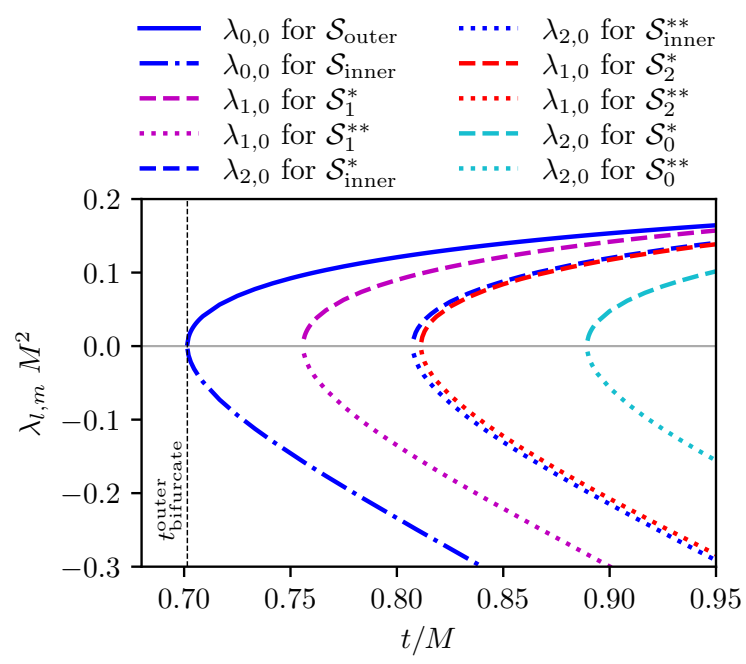

FIG. 5. Eigenvalues (with $m=0$ ) of the stability operator for the ten MOTSs participating in the five bifurcations shown in Fig. 2 . For each MOTS, we show the respective eigenvalue which tends to zero.

fully determining the deformation [33]. Intuitively $\Psi$ can be thought of as measuring the normal distance between the MOTS and its deformation.

Of particular interest is the eigenvalue spectrum $L_{\Sigma} \Psi=$ $\lambda_{l, m} \Psi$, where the $m=0$ eigenvalues correspond to axisymmetric deformations and in our non-spinning, axisymmetric case, all eigenvalues are real. $\lambda_{l, m}$ encodes information about geometry of the MOTS. Especially important is the principal (smallest) eigenvalue $\lambda_{0,0}$ [33-35]. If $\lambda_{0,0}$ is positive, then the MOTS is called strictly stable and evolves smoothly into the future. The world tubes traced out by such MOTSs are everywhere expanding and spacelike. Furthermore, these MOTSs act as barriers between trapped and untrapped surfaces in their vicinity. The vanishing of the principal eigenvalue has been identified with the bifurcation of $\mathcal{S}_{\text {outer }} / \mathcal{S}_{\text {inner }}[14,16,18]$.
We find these general considerations borne out in our work, though with a number of important modifications and caveats. First, of the potentially infinite number of MOTSs present in the interior of the merger, only three are strictly stable: $\mathcal{S}_{\text {outer }}, \mathcal{S}_{1}$ and $\mathcal{S}_{2}$. Only these surfaces act as barriers and they are also the only ones to have everywhere expanding space-like world tubes. These properties are precisely what one would associate with horizons. Therefore stability provides an unambiguous criterion by which the MOTSs corresponding to black hole boundaries may be identified. This observation underlies our choice of terminology for the $\mathrm{AH}$ advocated in this work: an $\mathrm{AH}$ is a stable MOTS $\left(\lambda_{0,0} \geq 0\right.$, i.e. we include the marginal case). All other MOTSs located have at least one negative eigenvalue.

We find that, associated to every bifurcation/annihilation is the vanishing of an eigenvalue of the stability operator - see Fig. 5 for the case of the bifurcations. The formation/annihilation of the AHs coincide with the vanishing of the principal eigenvalue, in line with the results described above. However, a key finding of our work is that, in the case that two MOTSs are unstable, it is the vanishing of one of the higher $m=0$ eigenvalues that coincides with their bifurcation/annihilation. A simple picture emerges: the MOTSs foliating a given world tube accrue an additional negative $m=0$ eigenvalue with each fold in time.

Finally, the properties of the spectrum provide further evidence for the evolution we have advocated in this letter. At a given bifurcation/annihilation event, we find that the numerical values of the eigenvalues of the surfaces involved connect smoothly (see again Fig. 5). This provides robust numerical evidence for the world tubes to be locally smooth across the bifurcations/annihilations shown in Fig. 2.

Summary. We have shown that the interior of a black hole merger is far richer than previously thought, containing a large (possibly infinite) number of hitherto unidentified MOTSs. These MOTSs were initially located using a new shooting method that sidesteps the drawback in exist- 
ing finders of requiring an initial guess for the surface of interest. The additional surfaces play a crucial role in the interior dynamics of the merger, and are responsible for the annihilation of the AHs of the original black holes. As such with these new MOTSs we reveal, for the first time, the full story of how two black holes become one, giving the analogue of the "pair of pants" diagram for the AH. The picture is considerably more complex than the equivalent picture for the event horizon and involves several world tubes that weave back and forth in time. The stability of MOTSs has played a clarifying role in our work. Rather than obscuring the utility of the quasi-local horizon framework, the multitude of MOTSs present during the merger actually highlights the rarity of stable MOTSs. Of all the MOTSs we have located, only three are stable, and these are precisely those that are most naturally associated with black hole boundaries. Moreover, we have found that associated to each bifurcation/annihilation event is the vanishing of some eigenvalue of the stability operator, not usually the principal one. This observation may aid in the analytical understanding of the world tubes traced out by unstable MOTSs.

Acknowledgements. We would like to express our gratitude to Graham Cox, Jose Luis Jaramillo, Badri Krishnan, Hari Kunduri and the members of the Memorial University Gravity Journal Club for valuable discussions and suggestions. IB was supported by the Natural Science and Engineering Research Council of Canada Discovery Grant 2018-0473. The work of RAH was supported by the Natural Science and Engineering Research Council of Canada through the Banting Postdoctoral Fellowship program and also by AOARD Grant FA2386-19-1-4077.

[1] S. W. Hawking and G. F. R. Ellis, The Large Scale Structure of Space-Time Cambridge Monographs on Mathematical Physics (Cambridge University Press, 1973).

[2] R. A. Matzner, H. E. Seidel, Stuart L. Shapiro, L. Smarr, W. M. Suen, Saul A. Teukolsky, and J. Winicour, "Geometry of a black hole collision," Science 270, 941-947 (1995).

[3] Olaf Dreyer, Badri Krishnan, Deirdre Shoemaker, and Erik Schnetter, "Introduction to isolated horizons in numerical relativity," Phys. Rev. D 67, 024018 (2003), arXiv:grqc/0206008.

[4] Badri Krishnan, "Fundamental properties and applications of quasi-local black hole horizons," Class. Quant. Grav. 25, 114005 (2008), arXiv:0712.1575 [gr-qc]

[5] Anshu Gupta, Badri Krishnan, Alex Nielsen, and Erik Schnetter, "Dynamics of marginally trapped surfaces in a binary black hole merger: Growth and approach to equilibrium,” Phys. Rev. D97, 084028 (2018), arXiv:1801.07048 [gr-qc]

[6] Luciano Rezzolla, Rodrigo P. Macedo, and Jose Luis Jaramillo, "Understanding the 'anti-kick' in the merger of binary black holes,' Phys. Rev. Lett. 104, 221101 (2010), arXiv:1003.0873 [gr-qc]
[7] Jose Luis Jaramillo, Rodrigo Panosso Macedo, Philipp Moesta, and Luciano Rezzolla, "Black-hole horizons as probes of black-hole dynamics I: post-merger recoil in head-on collisions," Phys. Rev. D 85, 084030 (2012) arXiv:1108.0060 [gr-qc].

[8] J. L. Jaramillo, R. P. Macedo, P. Moesta, and L. Rezzolla, "Towards a cross-correlation approach to strong-field dynamics in Black Hole spacetimes," AIP Conf. Proc. 1458, 158-173 (2012), arXiv:1205.3902 [gr-qc].

[9] Vaishak Prasad, Anshu Gupta, Sukanta Bose, Badri Krishnan, and Erik Schnetter, "News from horizons in binary black hole mergers," Phys. Rev. Lett. 125, 121101 (2020) arXiv:2003.06215 [gr-qc]

[10] Ishai Ben-Dov, "The Penrose inequality and apparent horizons," Phys. Rev. D 70, 124031 (2004), arXiv:grqc/0408066

[11] Ivan Booth, Lionel Brits, Jose A. Gonzalez, and Chris Van Den Broeck, "Marginally trapped tubes and dynamical horizons," Class. Quant. Grav. 23, 413-440 (2006), arXiv:grqc/0506119

[12] Sean A. Hayward, "Black holes: New horizons," in Recent developments in theoretical and experimental general relativity, gravitation and relativistic field theories. Proceedings, 9th Marcel Grossmann Meeting, MG'9, Rome, Italy, July 2-8, 2000. Pts. A-C (2000) pp. 568-580, arXiv:grqc/0008071 [gr-qc].

[13] Ishai Ben-Dov, "Penrose inequality and apparent horizons," Phys. Rev. D 70, 124031 (2004)

[14] Daniel Pook-Kolb, Ofek Birnholtz, Badri Krishnan, and Erik Schnetter, "Existence and stability of marginally trapped surfaces in black-hole spacetimes,"Phys. Rev. D 99, 064005 (2019)

[15] Daniel Pook-Kolb, Ofek Birnholtz, Badri Krishnan, and Erik Schnetter, "Interior of a binary black hole merger," Phys. Rev. Lett. 123, 171102 (2019)

[16] Daniel Pook-Kolb, Ofek Birnholtz, Badri Krishnan, and Erik Schnetter, "Self-intersecting marginally outer trapped surfaces," Phys. Rev. D 100, 084044 (2019).

[17] Daniel Pook-Kolb, Ofek Birnholtz, José Luis Jaramillo, Badri Krishnan, and Erik Schnetter, "Horizons in a binary black hole merger I: Geometry and area increase," (2020), arXiv:2006.03939 [gr-qc]

[18] Daniel Pook-Kolb, Ofek Birnholtz, José Luis Jaramillo, Badri Krishnan, and Erik Schnetter, "Horizons in a binary black hole merger II: Fluxes, multipole moments and stability," (2020), arXiv:2006.03940 [gr-qc].

[19] Ivan Booth, Robie A. Hennigar, and Saikat Mondal, "Marginally outer trapped surfaces in the Schwarzschild spacetime: Multiple self-intersections and extreme mass ratio mergers," Phys. Rev. D 102, 044031 (2020) arXiv:2005.05350 [gr-qc]

[20] Andrej Cadež, "Apparent horizons in the two-black-hole problem," Annals of Physics 83, 449 - 457 (1974)

[21] Ivan Booth, Robie A. Hennigar, and Daniel Pook-Kolb, "Ultimate fate of apparent horizons during a binary black hole merger I: locating and understanding axisymmetric marginally outer trapped surfaces," (2021).

[22] Daniel Pook-Kolb, Ofek Birnholtz, Ivan Booth, Robie A. Hennigar, José Luis Jaramillo, Badri Krishnan, Erik Schnetter, and Victor Zhang, "MOTS Finder version 1.5," (2021).

[23] Dieter R. Brill and Richard W. Lindquist, "Interaction en- 
ergy in geometrostatics," Phys. Rev. 131, 471-476 (1963).

[24] Frank Löffler, Joshua Faber, Eloisa Bentivegna, Tanja Bode, Peter Diener, Roland Haas, Ian Hinder, Bruno C. Mundim, Christian D. Ott, Erik Schnetter, Gabrielle Allen, Manuela Campanelli, and Pablo Laguna, "The Einstein Toolkit: A Community Computational Infrastructure for Relativistic Astrophysics," Class. Quantum Grav. 29, 115001 (2012), arXiv:1111.3344 [gr-qc]

[25] EinsteinToolkit, "Einstein Toolkit: Open software for relativistic astrophysics," http://einsteintoolkit. org//.

[26] Marcus Ansorg, Bernd Brügmann, and Wolfgang Tichy, "A single-domain spectral method for black hole puncture data," Phys. Rev. D 70, 064011 (2004), arXiv:grqc/0404056.

[27] J. David Brown, Peter Diener, Olivier Sarbach, Erik Schnetter, and Manuel Tiglio, "Turduckening black holes: an analytical and computational study," Phys. Rev. D 79, 044023 (2009) arXiv:0809.3533 [gr-qc]

[28] Sascha Husa, Ian Hinder, and Christiane Lechner, "Kranc: a Mathematica application to generate numerical codes for tensorial evolution equations," Comput. Phys. Commun. 174, 983-1004 (2006), arXiv:gr-qc/0404023

[29] Kranc, "Kranc: Kranc assembles numerical code," http: //kranccode.org//

[30] Miguel Alcubierre, Gabrielle Allen, Bernd Brügmann, Thomas Dramlitsch, Jose A. Font, Philippos Papadopou- los, Edward Seidel, Nikolaos Stergioulas, Wai-Mo Suen, and Ryoji Takahashi, "Towards a stable numerical evolution of strongly gravitating systems in general relativity: The Conformal treatments," Phys. Rev. D62, 044034 (2000). arXiv:gr-qc/0003071 [gr-qc].

[31] Miguel Alcubierre, Bernd Brügmann, Peter Diener, Michael Koppitz, Denis Pollney, Edward Seidel, and Ryoji Takahashi, "Gauge conditions for long term numerical black hole evolutions without excision,' Phys. Rev. D67, 084023 (2003), arXiv:gr-qc/0206072 [gr-qc]

[32] Daniel Pook-Kolb, Ivan Booth, and Robie A. Hennigar, "Ultimate fate of apparent horizons during a binary black hole merger II: Horizons weaving back and forth in time," (2021).

[33] Lars Andersson, Marc Mars, and Walter Simon, "Local existence of dynamical and trapping horizons," Phys.Rev.Lett. 95, 111102 (2005), arXiv:gr-qc/0506013 [gr-qc].

[34] Lars Andersson, Marc Mars, and Walter Simon, "Stability of marginally outer trapped surfaces and existence of marginally outer trapped tubes," Adv.Theor.Math.Phys. 12 (2008), arXiv:0704.2889 [gr-qc]

[35] Lars Andersson, Marc Mars, Jan Metzger, and Walter Simon, "The Time evolution of marginally trapped surfaces," Class. Quant. Grav. 26, 085018 (2009), arXiv:0811.4721 [gr-qc] 